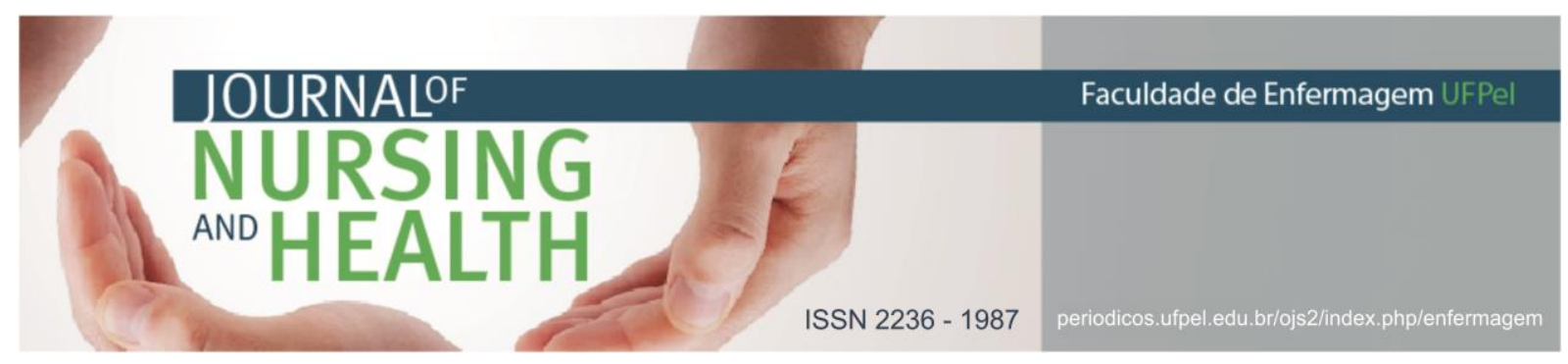

ARTIGO ORIGINAL

\title{
Prevalência de hanseníase em São Luís-Maranhão entre os anos de 2013 a 2015
}

\author{
Prevalence of leprosy in São Luís-Maranhão from 2013 to 2015 \\ Prevalencia de lepra en São Luís-Maranhão entre los años 2013 a 2015
}

Santos, Gerusinete Rodrigues Bastos dos ${ }^{1}$; Aragão, Francisca Bruna Arruda²; Brasil, Gabrielle Vieira da Silva3; Silva, Rodrigo Lopes da4; Garcês Júnior, Audivan Ribeiro5; Andrade, Luisa Marillac Ramos Lacerda de6; Lobão, Walder Jansen Mello de7; Penha, Lierbeth Santos Pereira8; Pereira, Joelmara Furtado dos Santos ${ }^{9}$; Batista, José Eduardo ${ }^{10}$

Como citar este artigo: Santos GRB, Aragão FBA, Brasil GVS, Silva RL, Garcês Júnior AR, Andrade LMRL, et al. Prevalência de hanseníase em São Luis-Maranhão entro os anos de 2013 a 2015. J. nurs. health. 2018;8(2): e188208

\section{RESUMO}

Objetivo: analisar e representar, espacialmente, a evolução da distribuição geográfica das ocorrências de Hanseníase no Maranhão entre os anos de 2013 a 2015, utilizando técnicas de geoprocessamento. Método: realizou-se análise, utilizando mapas e tabelas temáticas, com base nos registros do Sistema de Informação de Agravos de Notificação. Resultados: foram identificados 1.879 casos, somados entre 219 bairros residenciais. Para o ano de 2013, o distrito mais afetado foi o Tirirical; em 2014, aumentou a prevalência nos Distritos de Bequimão e Coroadinho e em 2015, houve uma redução de prevalências nos distritos de Tirirical Cohab, Coroadinho e Itaqui Bacanga. Conclusão: apesar de algumas localidades estudadas terem apresentado uma diminuição da prevalência entre 2013 e 2014, de 2014 a 2015 houve um aumento na quantidade de casos. 0 estudo de prevalências possibilitou essa identificação de casos, podendo ser observada a imprescindibilidade de subsidiar estratégias de controle.

Descritores: Hanseníase; Epidemiologia; Mapeamento geográfico.

1 Farmacêutica. Mestrado em Saúde do Adulto e da Criança. Universidade Federal do Maranhão (UFMA). E-mail: gerusinete@hotmail.com http: / / orcid.org/0000-0002-1602-4563

2 Enfermeira. Mestrado em Saúde do Adulto e da Criança. Universidade Federal do Maranhão (UFMA). E-mail: aragao_bruna@hotmail.com http://orcid.org/0000-0002-1191-0988

3 Nutricionista. Mestrado em Saúde do Adulto e da Criança. Universidade Federal do Maranhão (UFMA). E-mail: gabivieira40@gmail.com http://orcid.org/0000-0001-5849-8399

4 Médico. Mestrado em Saúde do Adulto e da Criança. Universidade Federal do Maranhão (UFMA). E-mail: rodrigo.1979@hotmail.com http://orcid.org/0000-0001-8489-159X

5 Geógrafo. Mestrado em Saúde e Ambiente. Universidade Federal do Maranhão (UFMA). E-mail: audivan@hotmail.com http://orcid.org/0000-0003-1047-6996

6 Médica. Especialista em Medicina do Trabalho. Universidade Federal do Maranhão (UFMA). E-mail: luisa.marillac.andrade@hotmail.com http://orcid.org/0000-0002-5788-6311

7 Dentista. Mestre em Ciências da Saúde. Universidade Federal do Maranhão (UFMA). E-mail: walderjanser@hotmail.com http://orcid.org/0000-0001-5839-5218

8 Biólogo. Universidade Estadual do Maranhão (UEMA). E-mail: liberth@gmail.com http://orcid.org/0000-00029508-7238

9 Enfermeira. Especialização em Saúde da Família. Universidade Federal do Maranhão. (UFMA). E-mail: joelmara7@hotmail.com http://orcid.org/0000-0002-4220-4437

10 Farmacêutico e Bioquímico. Doutor em Medicina Tropical e Saúde Pública. Universidade Federal do Maranhão (UFMA). E-mail: jbatista@gmail.com com http://orcid.org/0000-0003-2451-4789 


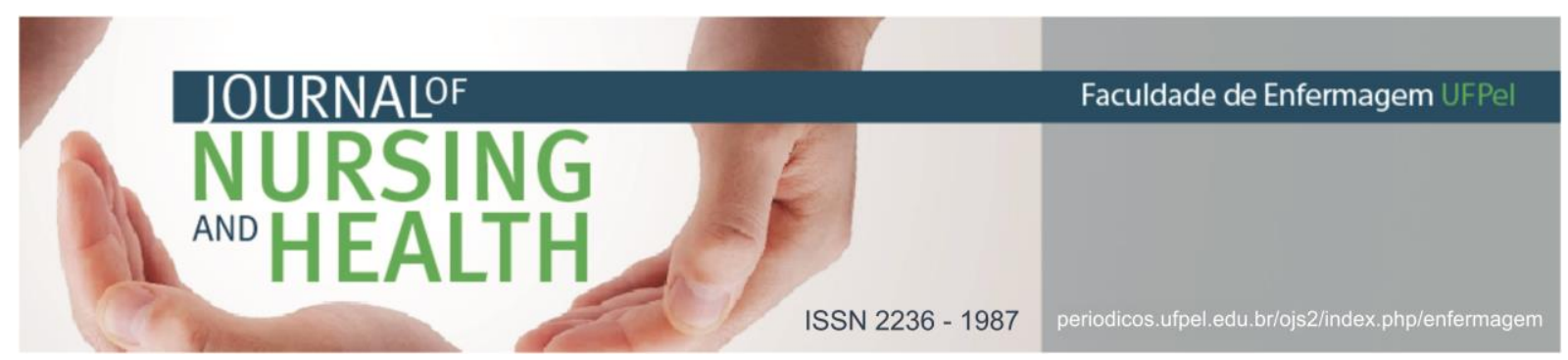

ABSTRACT

Objective: to analyze and spatially represent the evolution of the geographic distribution of occurrences of Leprosy in Maranhão between the years 2013 to 2015 using geoprocessing techniques. Method: the analysis was performed using maps and thematic tables, based on the records of the Notifiable Diseases Information System. Results: 1.879 cases were identified, among 219 residential districts. For the year 2013, the district most affected was Tirirical; in 2014, increased prevalence in the Districts of Bequimão and Coroadinho and in 2015, there was a reduction of prevalences in the districts of Tirirical Cohab, Coroadinho and Itaqui Bacanga. Conclusion: although some localities studied showed decrease in their prevalence between 2013 and 2014; there was increase in the number of cases from 2014 to 2015. The prevalence study made it possible to identify cases, and it is possible to observe the necessity of subsidizing control strategies

Descriptors: Leprosy; Epidemiology; Geographic mapping.

\section{RESUMEN}

Objetivo: analizar y representar, espacialmente, la evolución de la distribución geográfica de los sucesos de Lepra en Maranhão entre los años 2013 a 2015, utilizando técnicas de geoprocesamiento. Método: se realizó análisis, utilizando mapas y tablas temáticas con base en los registros del Sistema de Información de Agravios de Notificación. Resultados: se identificaron 1.879 casos, sumados entre 219 barrios residenciales. Para el año 2013, el distrito más afectado fue el Tirirical; en 2014, aumentó la prevalencia en los Distritos de Bequimão y Coroadinho y en 2015, hubo una reducción en los distritos de Tirirical Cohab, Coroadinho e Itaqui Bacanga. Conclusión: a pesar de que algunas localidades estudiadas presentaron una disminución la prevalencia entre 2013 a 2014, de 2014 a 2015 hubo un aumento en la cantidad de casos. El estudio cuantitativo de prevalencias posibilitó esa identificación de casos, pudiendo ser observada la imprescindibilidad de subsidiar estrategias de controle.

Descriptores: Lepra; Epidemiología; Mapeo geográfico.

\section{INTRODUÇÃO}

A hanseníase, conhecida popularmente pelo termo lepra, refere-se a uma patologia tão antiga quanto à humanidade. Desde que seguem os registros, na metade do século XVI, foi introduzida nas Américas pelos imigrantes europeus e depois, igualmente pelos escravos originados na África e, desde então, vem preocupando diversos países como o Brasil. ${ }^{1-2}$

Sua prevalência no Brasil ainda representa grandes desafios, considerando que, dentre todos os casos identificados nas Américas, apresenta aproximadamente $87 \%$, apontando para taxa mais elevada de prevalência e incidência do Continente
Sul-americano, sendo ainda, o segundo país no quantitativo de casos do mundo. ${ }^{3}$

Existe uma heterogeneidade na disseminação da doença, principalmente em razão das desigualdades socioeconômicas nas distintas regiões do país. ${ }^{4}$

O contágio da doença ocorre pela exposição da pessoa sadia em convivência familiar por tempo prolongado, apesar de existir grande probabilidade que seja gerada uma resistência natural ao bacilo Mycobacterium leprae 5 , que expõe pele e mucosas às lesões e manchas hipercrômicas ou hipocrômicas, podendo ainda impactar o sistema 


\section{JOURNALOF \\ NURSING \\ AND HEALTH}

nervoso periférico, cursando com perda da sensibilidade na localização das manchas e extremidades e, em casos mais graves, podem resultar em incapacidades. ${ }^{6}$

O bacilo é capaz de contaminar muitas pessoas, porém nem todas expressam a doença, por isso é conhecido como um microrganismo de alta infectividade e baixa patogenicidade. ${ }^{7}$ Quando não tratada, costuma afetar, em média, dois a três milhões de indivíduos no mundo, causando incapacidade física $^{8}$, o que tem representado um dos grandes desafios nos países endêmicos, pois apesar de ter havido melhora no controle, estes países continuam notificando casos novos que, em 2013, a Organização Mundial de Saúde (OMS) apresentou os coeficientes de identificação de novas incidências, em que foi possível verificar 126.913 casos novos na Índia e 31.044 no Brasil, sendo os países com as taxas de incidência mais elevadas. ${ }^{9} 0$ alto índice da doença tem apresentado uma estreita relação com os baixos Índices de Desenvolvimento Humano (IDH). ${ }^{10}$

Com o objetivo de combater e tentar eliminar a doença, o Brasil difundiu o Programa Nacional de Controle de Hanseníase (PNCH). ${ }^{11}$ Suas atividades deverão ser realizadas no âmbito da Atenção Básica, através de programações específicas de diagnóstico, para evitar casos de coprevalência. ${ }^{12}$

Independentemente da relevância da cadeia epidemiológica, as atividades associadas não são muito consideradas; quanto aos contatos dentro dos domicílios, estes configuram focos ocultos, que
ISSN 2236 - 198

alimentam a expressividade da epidemia na endemia hansênica. ${ }^{13}$

Conforme estudo recente ${ }^{14}$, a avaliação referente ao Programa de Controle de Hanseníase ( $\mathrm{PCH}$ ) no município de São Luís não tem apresentação atuação efetiva e resolutiva, pois o Programa apresenta deficiência no acompanhamento e monitorização dos casos, corroborando, assim, para que o município seja considerado hiperendêmico. A predominância em São Luís no ano de 2010 foi de 60,1 casos $/ 100.000$ habitantes, caracterizando um quadro de hiperendemicidade. No entanto, nos últimos seis anos observou-se uma redução significativa do percentual, atingindo 5,5 casos/100.000 habitantes ao ano, ou seja, uma patologia que ainda se apresenta como problemática social local. ${ }^{15}$

Diante do contexto abordado, o presente estudo tem como objetivo analisar e representar, espacialmente, a evolução da sua distribuição geográfica no Maranhão entre os anos de 2013 a 2015, utilizando técnicas de geoprocessamento.

\section{MATERIAIS E MÉTODOS}

Trata-se de um estudo retrospectivo, com abordagem quantitativa, realizado no período de setembro a outubro de 2016, com base nos registros de dados disponibilizados pelo Sistema de Informação de Agravos de Notificação (SINAN).

A população estimada de São Luís em 2016 foi de 1.082.935, com a medida de $834,785 \mathrm{Km}^{2}$ de área. ${ }^{16} \mathrm{O}$ município tem 211 bairros residenciais 


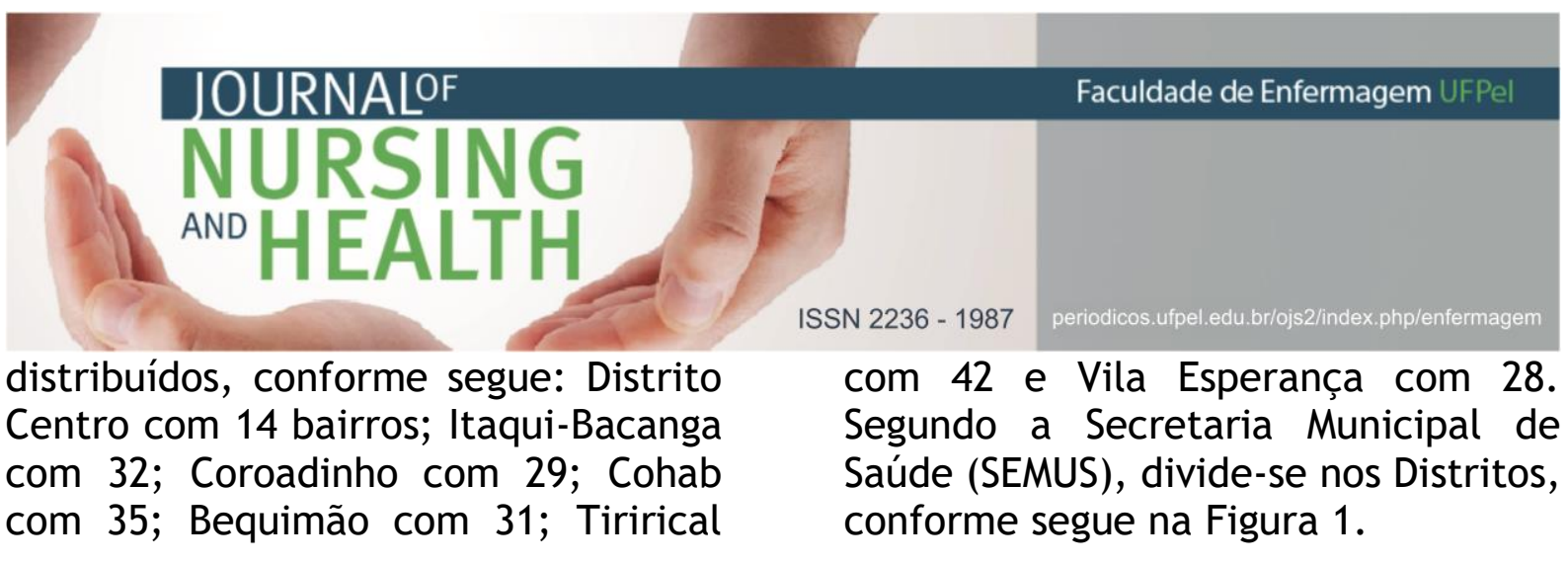

Figura 1: Distritos Sanitários de São Luís (MA), 2013-2015.

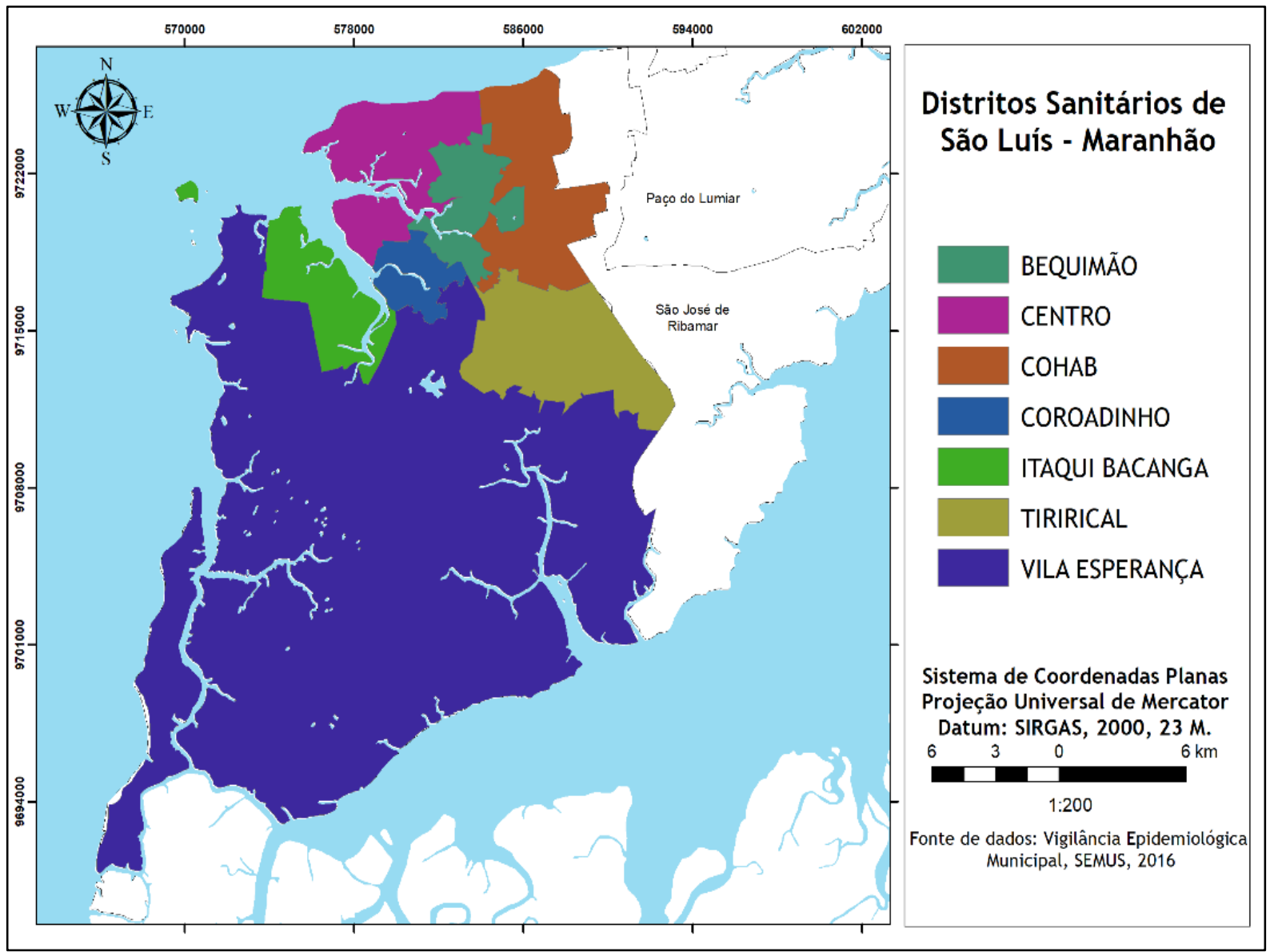

Fonte: SINAN/SEMUS, 2015.

Foram utilizados como dados secundários, o registro das notificações pertencentes ao Sistema de Informação de Agravos de Notificação (SINAN/Hanseníase) de São Luís entre 2013 e 2015, que foram autorizadas pela Secretaria Municipal de Saúde e pela Superintendência de Educação em Saúde de São Luís. Foram excluídas da análise, as fichas que se encontraram incompletas. Os dados foram agrupados em sete distritos sanitários, utilizando uma planilha eletrônica Microsoft Office Excel $2008 \AA$, para posterior georreferenciamento.

$\mathrm{Na}$ análise espacial e temporal do total de notificações foi utilizado o modelo de variação espacial discreta que, segundo o estudo ${ }^{17}$, possibilita a construção de uma aproximação 


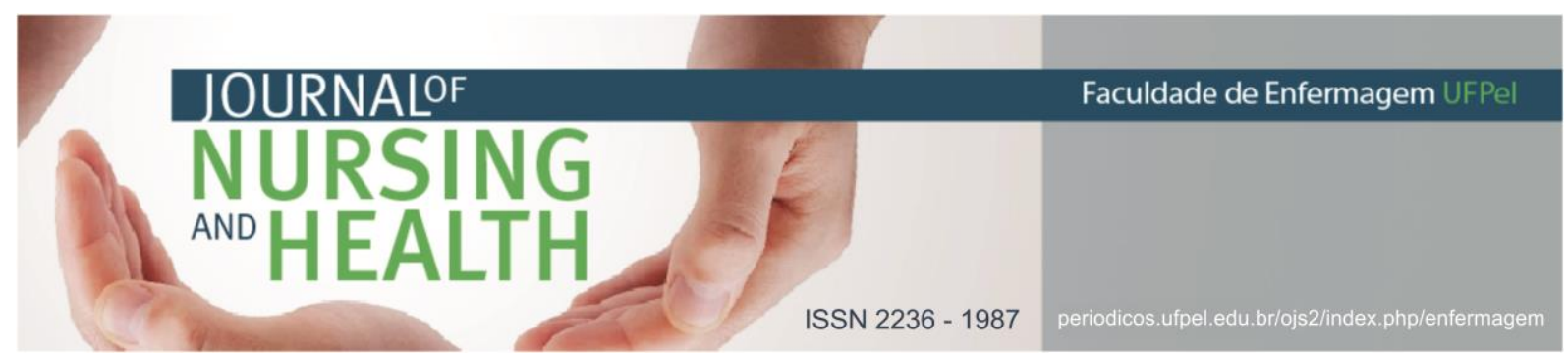

destinada à distribuição de um correspondentes aos anos de 2013, conjunto de variáveis aleatórias, avaliando sua distribuição. ${ }^{18}$ 2014 e 2015, entre todos os bairros residenciais de São Luís.

Os mapas temáticos foram confeccionados através do software ArcGis ${ }^{\circledR}$ na versão 10.1 , a base cartográfica utilizada foi do Instituto Brasileiro de Geografia e Estatística (IBGE), com projeção do SIRGAS 2000.

\section{RESULTADOS}

Através da análise proposta pelo

A Figura 1 representa a distribuição do consolidado de casos ano a ano, no período pesquisado.

A Figura 2 demonstra que em 2013 foram registrados 646 casos $(34,4 \%)$; em 2014 registrou-se 557 (29,6\%), e em 2015, 676 casos $(36,0 \%)$ de casos no período estudado. estudo, foram encontrados 1.879 casos

Figura 2 - Frequência por ano, diagnóstico dos Bairros Residenciais em São Luís (MA).

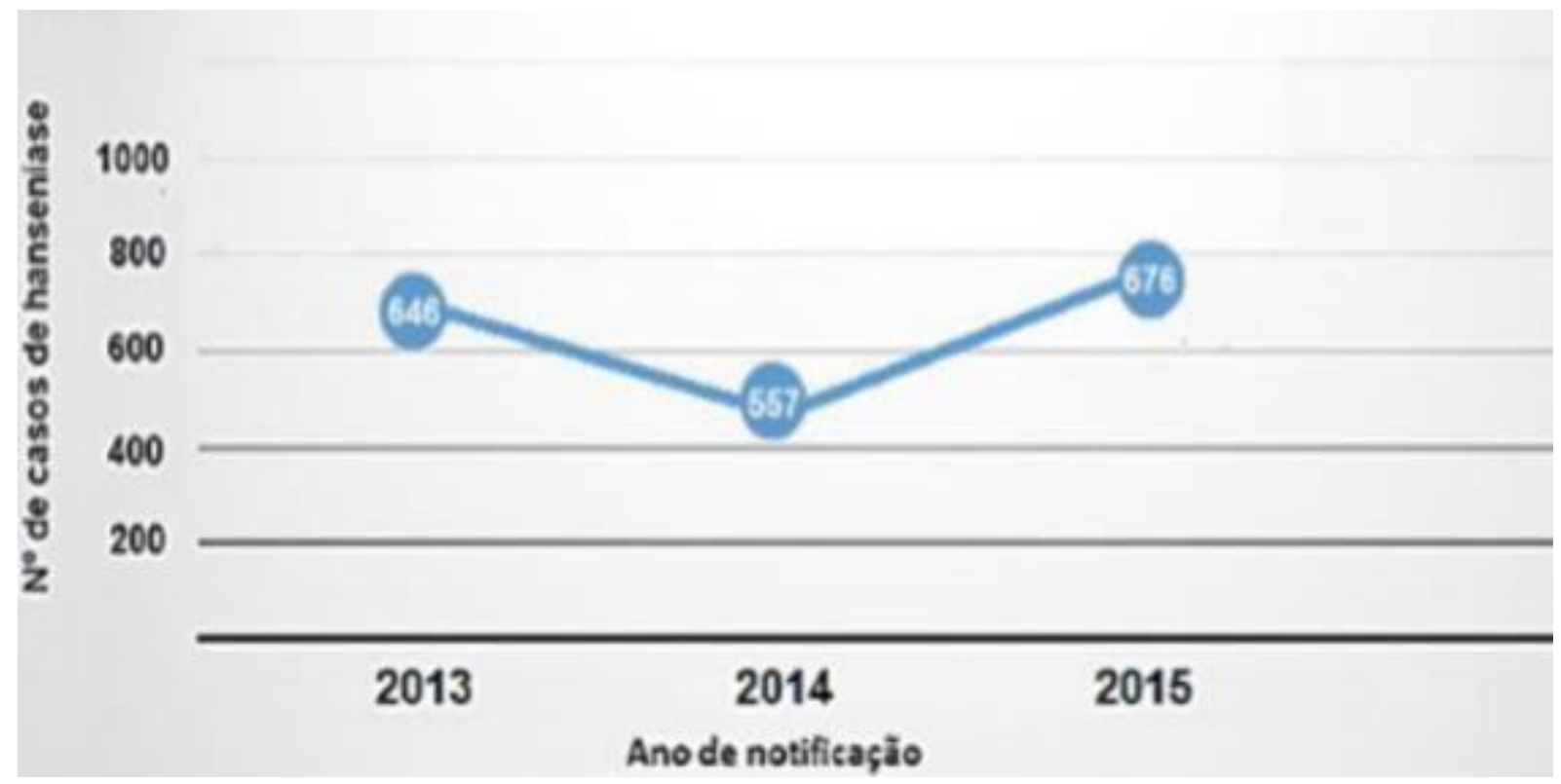

Fonte: SINAN/SEMUS, 2015

A Tabela 1 apresenta a distribuição anual de casos em cada distrito no período de 2013 a 2015. Em 2013, o Distrito Tirirical teve a maior prevalência, com 28,2\% ( $n=182)$, seguido do Distrito Cohab, o qual apresentou 16,9\% ( $n=109)$. Em 2014, o Distrito Itaqui Bacanga também apresentou maior prevalência, com $19,1 \% \quad(n=106)$, seguido do Distrito Tirirical com 25,2\% $(n=140)$. 0 ano de maior prevalência registrada foi em 2015, no Distrito Tirirical totalizando $20,7 \% \quad(n=205)$, seguido do Distrito Itaqui Bacanga com um percentual de $35,8 \%(n=354)$. 
Tabela 1: Distribuição anual de prevalência por Distritos Sanitários em São Luís (MA).

\begin{tabular}{lrrrrrr}
\hline \multicolumn{1}{c}{ DISTRITOS } & \multicolumn{2}{c}{2013} & & 2014 & \multicolumn{2}{c}{2015} \\
& $\mathbf{N}$ & $\%$ & $\mathbf{N}$ & $\%$ & $\mathbf{N}$ & $\%$ \\
Distrito Centro & 40 & 6,2 & 44 & 7,9 & 122 & 12,3 \\
Distrito Itaqui Bacanga & 125 & 19,3 & 106 & 19,1 & 354 & 35,8 \\
Distrito Coroadinho & 71 & 11,0 & 78 & 14,0 & 95 & 9,6 \\
Distrito Cohab & 109 & 16,9 & 97 & 17,4 & 117 & 11,8 \\
Distrito Bequimão & 51 & 7,9 & 36 & 6,5 & 33 & 3,3 \\
Distrito Tirirical & 182 & 28,2 & 140 & 25,2 & 205 & 20,7 \\
Distrito Vila Esperança & 68 & 10,5 & 55 & 9,9 & 64 & 6,5 \\
\hline Total de casos por distrito & 646 & 100,0 & 556 & 100,0 & 990 & 100,0 \\
\hline
\end{tabular}

Fonte: SINAN/SEMUS, 2015.

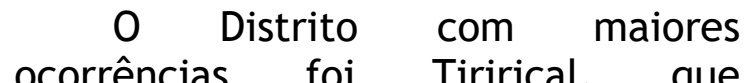
ocorrências foi Tirirical, que apresentou no bairro Vila Cidade Olímpica, em 2013, 48 casos; em 2014, 50 casos, e em 2015, 39 casos; no bairro Vila Cidade Operária, foram registrados 24 casos em 2013, 23 casos em 2014 e 38 casos em 2015. Contudo, nos demais distritos, as ocorrências foram: Itaqui Bacanga os bairros de maior prevalência foram Vila Nova que em 2013 registrou 14 casos, em 2015 mais 14 e 2016, 11 casos; e Vila Embratel que registrou a prevalência de 17 casos em 2013, 18 casos em 2014 e 27 casos em 2015 (Figura 3).

Para o ano de 2013, o Distrito mais afetado foi o Tirirical, seguido do Distrito Cohab e Itaqui Bacanga; em 2014, a prevalência aumentou no Distrito de Bequimão e Coroadinho. Já em 2015, as incidências no Tirirical, Cohab Coroadinho e Itaqui Bacanga foram reduzidas.

Nos anos de 2013 e 2014, a prevalência maior foi do sexo feminino, com $54,1 \%$ e $53,5 \%$ respectivamente.

Nos anos estudados, $86 \%(n=464)$ tinham a faixa etária de 15 anos ou mais em 2015, em 2014 foi a 90,5\% $(n=469)$ e em 2013, 87,5\% ( $n=487)$. Os pacientes no ensino médio em 2013 representaram 141 casos $(56,4 \%)$; em 2014 foram 131 casos (53,9\%); e em 2015 tiveram 159 casos (60,4\%).

Por fim da raça parda, foram identificados 354 (63,7\%) em 2013; em 2014 registraram $329(65,9 \%)$, e em 2015 foram evidenciados 395 (73,6\%), conforme a Tabela 2. 


\section{NURSING ${ }^{\text {ANO HEALTH }}$}

Figura 3: Distribuição espacial das ocorrências notificadas no Maranhão no período de 2013 a 2015.

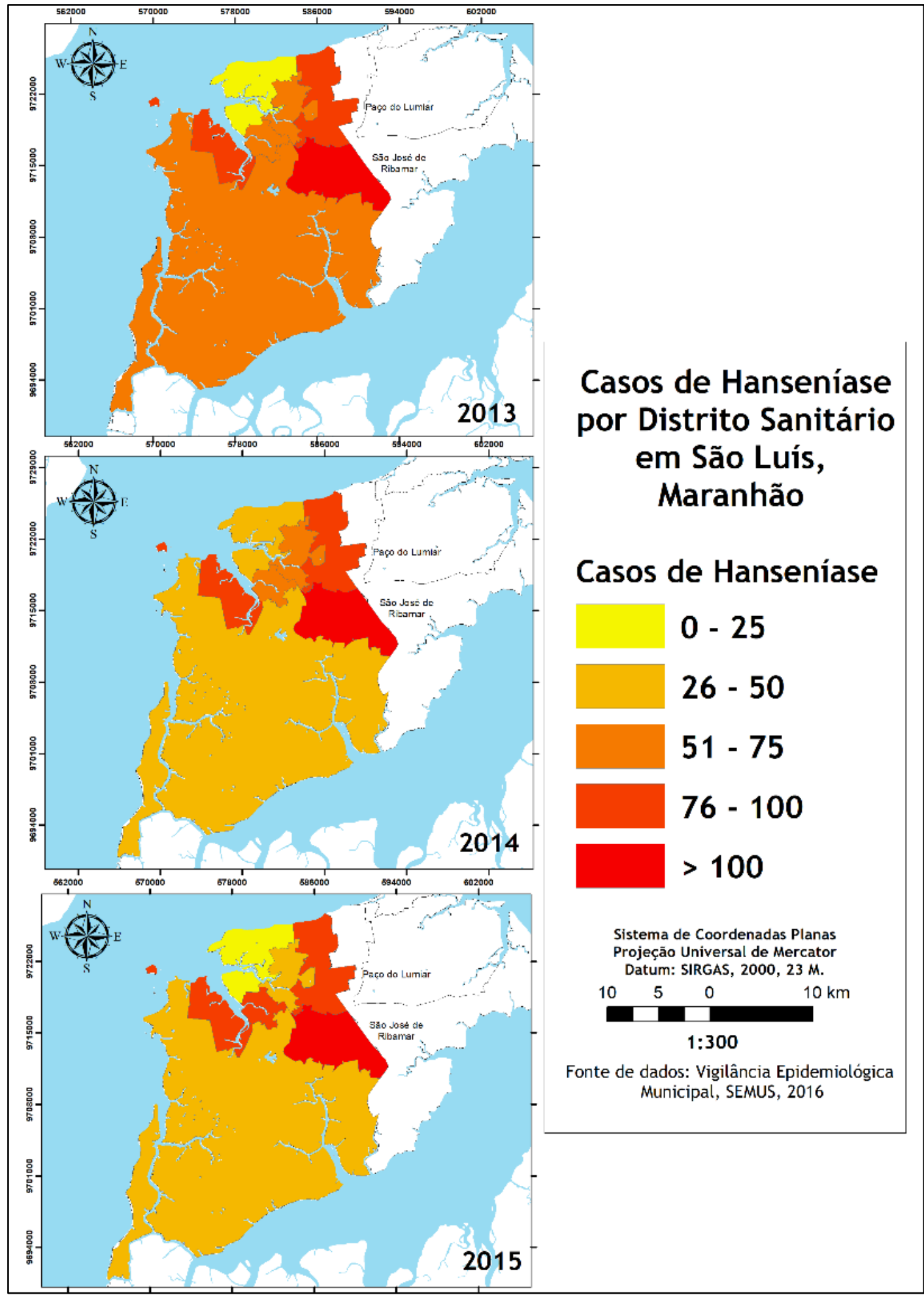

Fonte: SINAN/SEMUS, 2015. 


\section{NURSING \\ AND}

ISSN 2236 - 1987

Tabela 2 - Características sociodemográficas, Distritos de São Luís - MA, 2013-2015

\begin{tabular}{lcccccc}
\hline \multirow{2}{*}{ Variáveis } & \multicolumn{2}{c}{2013} & \multicolumn{2}{c}{2014} & \multicolumn{2}{c}{2015} \\
& N & $\%$ & N & $\%$ & N & $\%$ \\
Masculino & 256 & 45,9 & 241 & 46,5 & 272 & 50,7 \\
Feminino & 301 & 54,1 & 277 & 53,5 & 264 & 49,3 \\
Faixa etária & & & & & & \\
Oa14 anos & 70 & 12,5 & 49 & 9,5 & 72 & 13,4 \\
15 anos ou mais & 487 & 87,5 & 469 & 90,5 & 464 & 86,6 \\
Escolaridade & & & & & & \\
Analfabeto & 29 & 11,6 & 29 & 11,9 & 35 & 13,4 \\
Ensino Fundamental & 58 & 23,2 & 55 & 22,6 & 48 & 18,3 \\
Ensino Médio & 141 & 56,4 & 131 & 53,9 & 159 & 60,4 \\
Ensino Superior & 22 & 8,8 & 28 & 11,6 & 21 & 7,9 \\
Raça & & & & & & \\
Branca & 106 & 19,0 & 63 & 12,4 & 61 & 11,3 \\
Preta & 86 & 15,5 & 103 & 20,7 & 79 & 14,7 \\
Amarela & 10 & 1,8 & 5 & 1,0 & 1 & 0,4 \\
Parda & 354 & 63,7 & 329 & 65,9 & 395 & 73,6 \\
\hline
\end{tabular}

Fonte: SINAN/SEMUS, 2015.

A Tabela 3 demonstra que em 2013 foram registrados 211 casos identificados por encaminhamento (38,0\%); em 2014 foram 205(39,8\%), e em 2015 registraram 163 casos $(30,5 \%)$; os casos identificados por demanda espontânea foram registrados em 2013 com 313 casos $(56,4 \%)$, em 2014 com 284 (55,0\%) e em 2015 com 274 casos $(51,4 \%)$.

$\mathrm{Na}$ Tabela 4, os casos multibacilares registrados em 2013 foram $386(69,3 \%)$, em 2014 com 383 casos $(73,9 \%)$ e em 2015 com 404 casos
(75,4\%). Quanto à sua forma clínica, registraram 316 casos dimorfa em 2013 $(56,8 \%)$, em 2014 foram 310 casos $(59,9 \%)$ e em 2015 registraram 323 casos $(60,3 \%)$.

Quanto ao nível incapacitante no diagnóstico, o grau 0 em 2013 apontou 375 casos $(67,4 \%)$, em 2014 foram 331 casos $(63,9 \%)$ e em 2015 foram 368 casos $(68,7 \%)$. Referente ao grau 1 , em 2013 foram registrados 127 casos (22,9\%), em 2014 com 132 casos $(25,5 \%)$, e em 2015 foram 115 casos $(21,5 \%)$. 


\section{NURSING \\ ANO HEALTH}

ISSN 2236 - 1987

Tabela 3: Modo para detectar hanseníase em São Luís (MA) no período de 2013 a 2015.

\begin{tabular}{lcccccc}
\hline \multirow{2}{*}{ Modo de detecção } & \multicolumn{2}{c}{2013} & \multicolumn{2}{c}{2014} & \multicolumn{2}{c}{2015} \\
& $\mathbf{N}$ & $\%$ & $\mathbf{n}$ & $\%$ & $\mathbf{n}$ & $\%$ \\
\hline Encaminhamento & 211 & $38,0 \%$ & 205 & $39,8 \%$ & 163 & $30,5 \%$ \\
Demanda Espontânea & 313 & $56,4 \%$ & 284 & $55,0 \%$ & 274 & $51,4 \%$ \\
Exame Coletividade & 3 & $0,5 \%$ & 10 & $1,9 \%$ & 78 & $14,6 \%$ \\
Exame/Contatos & 27 & $4,9 \%$ & 16 & $3,2 \%$ & 18 & $3,4 \%$ \\
Outros & 1 & $0,2 \%$ & 1 & $0,1 \%$ & 1 & $0,1 \%$ \\
\hline
\end{tabular}

Fonte: SINAN/SEMUS, 2015.

Tabela 4: Características clínicas das incidências em São Luís (MA) no período de 2013 a 2015

\begin{tabular}{lcccccc}
\hline \multirow{2}{*}{$\begin{array}{l}\text { Variável } \\
\text { Classificação operacional }\end{array}$} & N & $\%$ & N & $\%$ & N & $\%$ \\
\hline Paucibacilar & 171 & $30,7 \%$ & 135 & $26,1 \%$ & 132 & $24,6 \%$ \\
Multibacilar & 386 & $69,3 \%$ & 383 & $73,9 \%$ & 404 & $75,4 \%$ \\
Forma clínica & & & & & & \\
Indeterminada & 54 & $9,7 \%$ & 37 & $7,1 \%$ & 39 & $7,2 \%$ \\
Virchowiana & 51 & $9,2 \%$ & 57 & $11,0 \%$ & 70 & $13,0 \%$ \\
Tuberculóide & 120 & $21,5 \%$ & 99 & $19,1 \%$ & 100 & $18,6 \%$ \\
Dimorfa & 316 & $56,8 \%$ & 310 & $59,9 \%$ & 323 & $60,3 \%$ \\
Não Classificada & 16 & $2,8 \%$ & 15 & $2,9 \%$ & 3 & $0,9 \%$ \\
Grau de incapacidade no diagnóstico & & & & & \\
Grau 0 & 375 & $67,4 \%$ & 331 & $63,9 \%$ & 368 & $68,7 \%$ \\
Grau 1 & 127 & $22,9 \%$ & 132 & $25,5 \%$ & 115 & $21,5 \%$ \\
Grau 2 & 35 & $6,2 \%$ & 31 & $5,9 \%$ & 30 & $5,5 \%$ \\
Não avaliado & 20 & $3,5 \%$ & 24 & $4,7 \%$ & 23 & $4,3 \%$ \\
\hline
\end{tabular}

Fonte: SINAN/SEMUS, 2015.

\section{DISCUSSÃO}

A hanseníase merece uma atenção especial no que se refere ao controle e ao acompanhamento longitudinal e integral do doente e seus familiares. A região nordeste é a terceira região com maior prevalência por 100 mil habitantes $(23,79 / 100 \mathrm{mil}$ habitantes), sendo classificada como muito alto. ${ }^{19} \mathrm{Em} \mathrm{2013}$, o coeficiente de 


\section{JOURNALOF \\ NURSING \\ AND HEALTH}

ISSN 2236 - 1987

descrever o perfil epidemiológico intradomiciliares em Buriticupu - MA, cujo objetivo foi identificar 294 contatos, com um questionário sobre a situação socioeconômica e demográfica e formulário padronizado destinado ao exame físico. Predominaram os solteiros $(68,7 \%)$, feminino $(54,1 \%)$ e, dentre todas as outras variáveis identificadas, foi concluído que as precárias condições socioeconômicas e ambientais ligadas aos cuidados epidemiológicos possivelmente corroboram para sua progressão. Segundo dados de 2009 no Brasil, 67,5\% dos casos identificados possuíam escolaridade fundamental incompleto e $11,7 \%$ destes eram analfabetos. ${ }^{5}$

Segundo os autores ${ }^{4}$, ao estudarem suas características epidemiológicas e espaciais entre 2001 a 2012, verificaram que dos 54.719 novos casos notificados, a maioria era formada por homens entre 20 a 39 anos. Neste estudo, o município com maior prevalência foi a capital do Estado, com os maiores coeficientes de detecção $(23,27$ casos $/ 10 \quad \mathrm{mil}$ habitantes) foi registrado em Junco do Maranhão.

\section{CONCLUSÃO} estão sendo utilizadas as tecnologias de geoinformação para dar suporte às análises ambientais, auxiliando na visualização de ocorrências na saúde pública. ${ }^{3}$ A capacidade de integração de informações dos Sistemas de Informações Geográficas (SIGs) torna mais eficaz, sintetizando, acelerando e automatizando a análise epidemiológica da situação. ${ }^{5}$

Foi realizado um estudo 9 transversal, com a finalidade de
A capital do Maranhão apresentou um alto encadeamento de transmissibilidade da hanseníase. Os apontados como principais elos nessa rede de transmissão; no município local do estudo, a pesquisa indicou um período prolongado de convívio dos casos antes do início do tratamento com seus respectivos contatos. Este estudo foi de relevância, possibilitando contatos domiciliares dos índices são 


\section{JOURNALOF \\ NURSING \\ AND HEALTH}

ISSN 2236 - 198

não puderam ser avaliadas, devido à ausência de algumas informações nas mesmas.

\section{REFERÊNCIAS}

1 Gomes MP, Pinto MH, Nardi SMT, Paschoal VDA. Hanseníase: a visão do profissional da saúde no discurso do sujeito coletivo. Rev bras pesqui saúde [Internet]. 2014[acesso em 2018 set 25];16(1):41-8. Disponível em: periodicos.ufes.br/RBPS/article/downlo $\mathrm{ad} / 8485 / 5981$

2 Alves ED, Ferreira TL, Ferreira IN. Hanseníase: avanços e desafios. Brasília: Nesprom; 2014:492.

3 Araújo LS, Santiago IS, Araújo OD, Araújo TMEA, Almeida PD, Sena IVO. Ações de enfermagem na prevenção e controle da hanseníase: uma revisão integrativa. Rev enferm UFPI [Internet]. 2016[acesso em 2018 set 25];5(2):69-74. Disponível em: http://www.ojs.ufpi.br/index.php/re ufpi/article/view/4655/pdf

4 Barbosa DR, Almeida MG, dos Santos AG. Características epidemiológicas e espaciais da hanseníase no Estado do Maranhão, Brasil, 2001-2012. Medicina (Ribeirão Preto) [Internet]. 2014[acesso em 2018 set 25];47(4):347-56. Disponível em: http://revista.fmrp.usp.br/2014/vol4 7n4/REV_Caracteristicasepidemiologicas-e-espaciais-dahanseniase-no-Estado-do-

Maranhao.pdf

5 Pacheco MAB, Aires MLL, Seixas ES. Prevalência e controle de hanseníase: pesquisa em uma ocupação urbana de São Luís, Maranhão, Brasil. Rev bras med fam comunidade [Internet]. 2014[acesso em 2018 set 25];9(30):23- 


\section{JOURNALOF \\ AND}

NURSING
30.

Disponível

em:

https://www.rbmfc.org.br/rbmfc/arti cle/view/690

6 Ministério da Saúde (BR). Secretaria de Vigilância em Saúde: guia prático sobre hanseníase. Brasília; 2017.

7 Pinheiro MGC, Silva SYB, França ALM, Monteiro BR, Simpson CA. Hanseníase: uma abordagem educativa com estudantes do ensino médio. Rev pesqui cuid fundam [Internet]. 2014[acesso em 2018 set 24]:776-84. Disponível em: http: / /www.redalyc.org/html/5057/5 05750622032/

8 Cássia Ribeiro G, Fabri ACOC, Amaral EP, Machado ÍE, Lana FCF. Estimativa da prevalência oculta da hanseníase na microrregião de Diamantina-Minas Gerais. Rev eletrônica enferm [Internet]. 2014[acesso em 2018 set 25];16(4):728-35. Disponível em: https://www.fen.ufg.br/revista/v16/ n4/pdf/v16n4a04.pdf

9 Palit A, Inamadar AC. Childhood leprosy in India over the past two decades. Lepr rev. 2014;85(2):93-9.

10 Fernandes RZS, Gouveia Vilela MF. Estratégias de integração das práticas assistenciais de saúde e de vigilância sanitária no contexto de implementação da Rede Cegonha. Ciênc saúde colet [Internet]. 2014[acesso em 2018 set 25];19(11):4457-66. Disponível em: http: / / www.scielo.br/pdf/csc/v19n11 /1413-8123-csc-19-11-4457.pdf

11 Lanza FM, Vieira NF, Oliveira MMC, Lana FCF. Avaliação da atenção primária no controle da hanseníase: proposta de uma ferramenta destinada aos usuários. Rev esc enferm USP [Internet]. 2014[acesso em 2018 set
ISSN 2236 - 1987

25];48(6):1054-61. Disponível em: http: / / www. scielo.br/pdf/reeusp/v48 n6/pt_0080-6234-reeusp-48-061054.pdf

12 Savassi LCM, Moderna CM. Hanseníase e a atenção primária: desafios educacionais e assistenciais na perspectiva de médicos residentes. Hansen int [Internet]. 2015[acesso em 2018 set 25];40(2):2-16. Disponível em: http://www.ilsl.br/revista/detalhe_a rtigo.php?id=12359

13 Ministério da Saúde (BR). Agência Nacional de Vigilância Sanitária. Portaria $n^{\circ}$. 149, de 3 de fevereiro de 2016. Aprova as Diretrizes para vigilância, atenção e eliminação da hanseníase como problema de saúde pública, com a finalidade de orientar os gestores e os profissionais dos serviços de saúde. Brasília; 2016.

14 Goiabeira YNLA, Rolim ILTP, Aquino DMC, Santos LH, Lima ABS, Soeiro VMS. Programa de controle da hanseníase em capital hiperendêmica: uma avaliação operacional. Rev baiana enferm [Internet]. 2018[acesso em 2018 set 25];32:e25144. Disponível em: https: / / portalseer.ufba.br/index.php /enfermagem/article/view/25144/15 808

15 Pacheco MAB, Aires MLL, Seixas ES. Prevalência e controle de hanseníase: pesquisa em uma ocupação urbana de São Luís, Maranhão, Brasil. Rev bras med fam comunidade [Internet]. 2014[acesso em 2018 set 25];9(30):23$30 . \quad$ Disponível em: https: //www.rbmfc.org.br/rbmfc/arti cle/view/690/602

16 Brasil. Instituto Brasileiro de Geografia e Estatística (IBGE). Projeção da população de São Luiz 


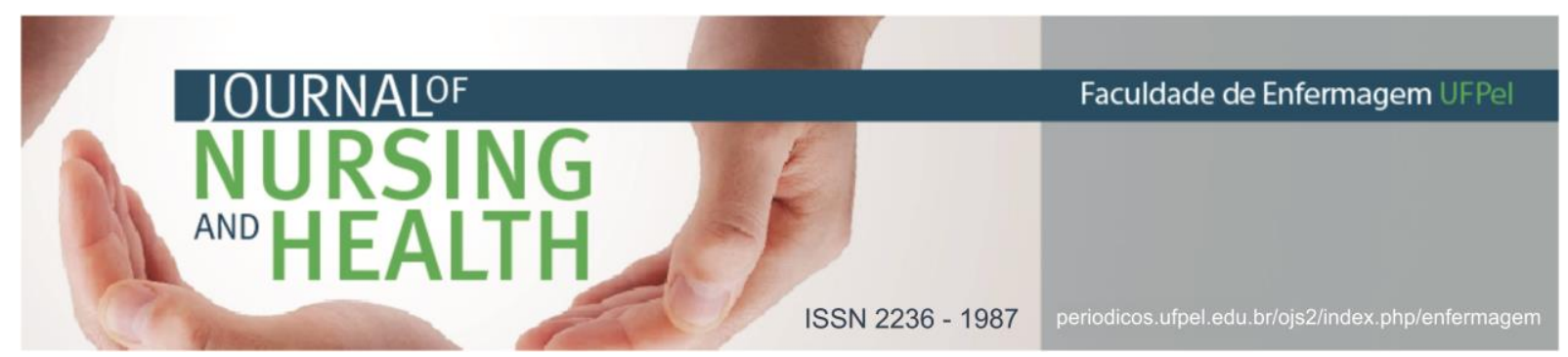

[Internet]. Rio de Janeiro, 2016[acesso 2018 set 27. Rio de Janeiro, IBGE, 2016. Disponível em: https://cidades.ibge.gov.br/brasil/ma /sao-luis/panorama

17 Alencar CH, Ramos AN, Santos ES, Richter J, Heukelbach J. Clusters of leprosy transmission and of late diagnosis in a highly endemic area in Brazil: focus on different spatial analysis approaches. Trop med int health [Internet]. 2012[cited 2018 Sept 25];17(4):518-25. Available from: https://onlinelibrary.wiley.com/doi/a bs/10.1111/j.1365-3156.2011.02945.x

18 Druck S. Análise espacial de dados geográficos: Empraba Cerrados; 2004.

19 Gonçalves NV, Araujo EN, Júnior S, Silva A, Pereira WMM, Miranda CSC, et al. Distribuição espaço-temporal da leptospirose e fatores de risco em Belém, Pará, Brasil. Ciênc saúde colet [Internet]. 2016[acesso em 2018 set 25];21:3947-55. Disponível em: http: / / www.scielo.br/pdf/csc/v21n12 /1413-8123-csc-21-12-3947.pdf

20 Araújo AERA, Aquino DMC, Goulart IMB, Pereira SRF, Figueiredo IA, Serra $\mathrm{HO}$, et al. Complicações neurais e incapacidades em hanseníase em capital do nordeste brasileiro com alta endemicidade. Rev bras epidemiol [Internet]. 2014[acesso em 2018 set 25];17:899-910. Disponível em: http://www.scielo.br/pdf/rbepid/v17 n4/pt_1415-790X-rbepid-17-0400899.pdf 